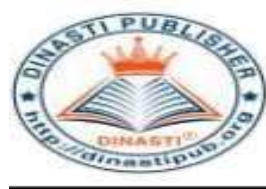

\title{
HARRY POTTER" AND MORAL VALUES LEARNING: A QUALITATIVE STUDY OF THE RESPONSE OF CHILDREN AGED 11-13 YEARS AGAINST J.K. ROWLING BOOKS
}

\author{
Ratna Setyowati Putri ${ }^{1}$, Rosma indriana Purba ${ }^{2}$, Donna Imelda ${ }^{3}$ \\ ${ }^{1)}$ Pelita Harapan University, Jakarta, Indonesia \\ ${ }^{2)}$ Pelita Harapan University, Jakarta, Indonesia \\ ${ }^{3)}$ Pelita Harapan University, Jakarta, Indonesia
}

\begin{tabular}{|c|c|}
\hline $\begin{array}{l}\text { ARTICLE INFORMATION } \\
\text { Received: } 1 \text { February } 2020 \\
\text { Revised: } 7 \text { February } 2020 \\
\text { Issued: } 11 \text { February } 2020 \\
\text { Corresponding author: } \\
\text { Ari Prayogo } \\
\text { E-mail: } \\
\text { catnasetyowatiputri@gmail.com } \\
\text { ourba.rosma@gmail.com } \\
\text { deimelda18@gmail.com }\end{array}$ & $\begin{array}{l}\text { Abstract: "Harry Potter" and Moral Value Learning } \\
\text { is a qualitative study of the response of students aged } \\
11-13 \text { at an international Christian school in Bekasi, } \\
\text { Indonesia, towards the phenomenal J.K Rowling text. } \\
\text { Through the hermeneutic method, this research tried } \\
\text { to seek the influence of Harry Potter texts, which are } \\
\text { suspected of bringing adverse effects for their young } \\
\text { readers. Besides, this research offers rational } \\
\text { considerations to the authority in schools, especially } \\
\text { regarding matters related to book censorship. Three } \\
\text { things investigated in this study were the ability of } \\
\text { students to think critically in making a distinction } \\
\text { between fiction and facts in the Harry Potter text, the } \\
\text { influence of the Harry Potter text on students' } \\
\text { tendencies of violence and occultism, and finally } \\
\text { about student learning of moral values contained in } \\
\text { the text. The data was collected through in-depth } \\
\text { interviews with two male respondents and three } \\
\text { female respondents aged } 11-13 \text { years with a high } \\
\text { religious background, mediocre, and less significant } \\
\text { religious background. It was found that all } \\
\text { respondents were able to distinguish between fiction } \\
\text { and facts in the Harry Potter texts, and there was no } \\
\text { significant evidence that the texts influenced } \\
\text { respondents' tendency for violence nor respondents' } \\
\text { interest in occultism. This study also found that } \\
\text { respondents were able to identify the moral values } \\
\text { contained in the text. } \\
\text { Keywords: Moral, Values, Harry Potter, Violence, } \\
\text { Occultism }\end{array}$ \\
\hline
\end{tabular}




\section{INTRODUCTION}

Education is a fundamental action that touches the roots of our lives, changes, and determines human life. Education is humanizing young people. Education is a form of living in a community that brings young people to the level of full-fledged human beings (Driyarkara, 1991). Moral education is one of the significant components of education. Unfortunately, educational practices are suspected not to support the achievement of the whole human being fully. In schools with standardized tests waiting at the end of the school year, educational practices tend to prioritize the transfer of knowledge (cognitive and psychomotor) and forget the learners' affective aspects. With so many curriculum agendas to prepare students for exams, moral education, which should occupy a key position in education, could be the last agenda in educational practice. Educators tend to "catch up" by giving a list of moral values to apply in life, asking students to memorize, training students to identify and answer multiple-choice questions, while every educator will agree that instilling values like this is not enough.

In the class that has entered the next stage, simple examples of real-life applications accompany the discussion of these moral values. Usually, the examples show clearly that Mr. So-and-so shows this or that moral value. The black and white border, or good and bad, is distinct. Meanwhile, in the real world, black and white boundaries are often blurred. The real problems that students face in their lives are not as simple as the examples given in class. The list of moral values that they memorized does not help them in real life. There is a gap here.

With global phenomena and cultures increasingly dominating contemporary life, students need to be equipped with critical thinking skills to respond to the various choices existing in various aspects of life. These critical thinking skills need to be built in context, not in isolation. Students need to face and analyze world problems to demonstrate their understanding of the theory and knowledge they possess. In this case, Harry Potter books with the controversy underlying them, the setting of the two worlds they display, their characterizations, and their exciting plots provide materials for criticism appropriate to the interests and development of students. Harry Potter has become part of contemporary culture, and so instead of intercepting students' interest in devouring this book series due to the controversy that follows the series, is there room to use this Potter as moral education material for students?

In one and many ways, a book influences and changes the reader. An inspiring book inspires its readers. Melancholy books make their readers feel melancholy too. Furthermore, if books affect their readers, how do Harry Potter books affect their readers? Do they see the books as fictional magic or a real occult practice? To what extent does Harry Potter text influence the orientation of its readers' value? This research sought to observe the extent of the influence of Harry Potter books on the respondents.

\section{LITERATURE REVIEW}

\section{Research question}

Assuming that a book influences its reader and that the reader is an individual who actively responds to and interprets the text, the researcher intends to investigate the following:

a. To what extent is the influence of Harry Potter books on students' critical thinking skills to distinguish between fiction and facts?

b. How far is the influence of Harry Potter books on the students' tendency to commit acts of violence and/ or sympathize with the occult?

c. To what extent does the Harry Potter book inspire moral values to students? 


\section{Research Hypothesis}

The fictional text in this study, Harry Potter text, is a text that can be multiinterpreted, not only related to the occult but also the learning of moral values contained therein.

This research hypothesis refers to Piaget's opinion that at the age of 7-12 years, children have shown a remarkable capacity for logical reasoning. In the next stage, at the age of 12 years and beyond, an individual can think logically, formulate hypotheses, and by considering all related essential facts and ideas, he/she can make conclusions correctly. Because the respondents of this study were children aged 11-13 years, the respondents are likely able to distinguish between fiction (fantasy) and fact (reality). Children reading Harry Potter can understand that the story is an unreal fiction, although the narration contains facts related to history as the setting and characters of the story which makes the story is more vivid and sounds real. The ability to make the distinction between fiction and fact becomes vital to respond to Plato's mimesis theory. Plato stated that readers can be influenced and can imitate what they read. Before deciding to emulate what he reads, the reader who can differentiate between fiction and fact will make further considerations whether what he is dealing with is just a fantasy or reality that can happen in real life.

Edmund M. Kern's opinion underlies the second hypothesis. Kern argues that a reader is not a passive subject who accepts without processing the narration and information conveyed by the reading text. Children reading Harry Potter books are not only captivated and blindly follow what is in the story. Thus, regarding the occult that is the controversy of this story, it is assumed that children reading Harry Potter books can distinguish that what Harry Potter story describes is a fiction. Besides, Matlin's top-down theory or pre-conception provides a rationale that respondents who have never known the occult will not be able to identify occultism in the text.

Furthermore, regarding the violence displayed in the text, it is predicted that the violence exposed in the text is not enough to influence respondents' tendency for violence.

Thirdly, based on Kern's opinion about children's ability to judge things, to choose the good from the bad, it is predicted that children reading Harry Potter books can judge whether the characters described in the story are good or bad characters and are able to recognize the moral dimension of the Harry Potter story. Readers of Harry Potter books who come from families with a stronger Christian background will be better able to link the main points in the Harry Potter story with Christian teachings. Conversely, readers who have weaker Christian backgrounds will see it as a moral value in general.

Following Piaget's theory of moral development, the research respondents are at the end of the third stage and the beginning of the last stage in their moral development. In this stage, respondents begin to take into account subjective considerations such as one's intention in moral judgment. The existence of moral reasoning also marks this stage. Hence, in their moral assessment of the Harry Potter text, the respondents may defend their assessment of the moral dilemma they encounter by considering the intentions of the characters or giving other moral reasons.

Thus, the research hypothesis is formulated as follows. First, respondents can distinguish between fiction and reality. Secondly, respondents are not affected by violence and occultism, even if it is in the Harry Potter texts. Finally, respondents can recognize and learn moral values from the characters in the Harry Potter texts. 


\section{Research purposes}

This study aims to respond to the concerns with the stigma of particular books in the sense of tabooing these books without ever reading them and researching them further. This kind of taboo bases its position on Plato's opinion of mimesis, namely that the reader imitates what he reads. By examining the controversial Harry Potter text suspected of causing adverse effects on its readers, this research seeks to reveal how far Plato's mimesis is proven.

This study also aims to investigate whether respondents can think critically in distinguishing fiction and facts, to investigate the influence of the Harry Potter texts on the tendencies of violence and occultism, and to investigate the extent to which the Harry Potter texts inspire children to moral values.

In the end, this research intends to open a room for negotiations with schools' authorities and families regarding similar books. The banning of literary work needs to be indepth research based, instead of judgemental feeling only.

\section{Theoretical basis}

This study uses the opinions of several experts, namely Piaget's theory of cognitive development, Piaget's theory of moral development, the theory of perceptions proposed by Matlin, and Kern and Bettleheim's theories regarding the effect of a reading text on its readers.

Firstly, Piaget with cognitive development theory provides a rationale for the stages of development that occur in children aged 11-13 who are the respondents of this study; in this case, the respondents are at the end of the concrete operational stage and the beginning of the formal operational stage (Rice, 1998). At the concrete operational stage, children show a high capacity to think logically at a concrete level, whereas at the formal operational stage, children can use logic and abstract concepts separately from his concrete objects. As the respondents are at the end of the concrete operational stage and the beginning of the formal operational stage, it is assumed that the respondents are very capable of thinking logically at a concrete level but are still at an early stage in using logic and abstract concepts separately from the concrete objects. Respondents have also begun to be able to think about ideas, think beyond facts in the direction of something that might happen, and form hypotheses. When connected with this research, based on these conditions, respondents should be able to distinguish between facts and fiction in the Harry Potter text. This study is to prove the respondents' ability in that area.

Secondly, the same expert's opinion, Piaget's, is used to see the development of respondents' moral judgment. The description of Piaget's stages of moral development is classified based on the stages of cognitive development. In other words, moral judgment develops with age (Atkinson, 1996). In this study, his opinion is used to find out the extent of respondents' moral development so that the ability of respondents to understand moral values in the text can be predicted.

Thirdly, Bettelheim (1976) and Kern (2006) share the same opinion that a reading text affects its reader. According to Kern, readers are not subjects who passively accept what is written. Even young readers can process the text they read. Based on Bettelheim and Kern's thoughts and the controversy over the Harry Potter texts, this research will investigate the extent to which the Harry Potter texts influence the respondents' tendencies towards violence and the occult. Besides, the research will examine the influence of the texts on the respondents' value orientation. Bettelheim believes that fairy tales provide moral values ready to be captured by respondents. This research will examine whether the respondent can "catch" the values, either positive or negative values, in the Harry Potter texts. 
Finally, Matlin's (1994) opinion on the process of perception will be used in this study to explain whether the respondents see the Harry Potter text as an occult fact or something else. This concept of perception explains why two people who see the same thing can have two different opinions about it.

\section{RESEARCH METHODS}

This research is a qualitative study using hermeneutics research (Nasution, 1998; Hardiman, 1992) as a means of interpreting the data collected.

The method is selected based on the purpose of the study, namely, describing how readers of the Harry Potter text interpret the text: as fiction that tells the story of magic or the reality of occult practices that attract them to study occultism further. This method also allows researchers to find the extent of the influence of Harry Potter texts on the value orientation of the respondents.

Research subject

This study applies a purposive sample (Fraenkel, 1993) by selecting five students aged 11 to 13 years and attending an international Christian school in Bekasi, Indonesia, as the primary respondents. Sampling with the age range above is based on the consideration that the Harry Potter text is under chapter books category, i.e., a book consisting of several chapters with very minimal story illustrations, which poses too high reading difficulty level for children under ten years old. Besides, this age selection is also based on readers' age recommended by Scholastic for the 5th to 7th Harry Potter book series.

The profiles of children selected as respondents are those aged 11-13 years, who have or have been reading more than one of the Harry Potter books. Respondents are from families with Christian backgrounds under the following three categories, with at least one respondent representing each category:

1. a strong Christian background, meaning that the respondent lives in a Christian family that puts a strong emphasis on the formation of Christian values in the lives of the respondent and encourages the application of Christian values in daily life.

2. a mediocre Christian background, meaning that the respondent's family is Christian, encourages Christian values but does not demand the application in daily life.

3. weak Christian backgrounds, meaning that the respondent comes from a Christian family, but there has never been a discussion about Christian values in the family, Christianity is more as a routine.

\section{Research Instruments}

The research instruments used in this study are:

1. A series of texts taken from the Harry Potter texts that have been read by respondents.

Given the fact that the Harry Potter books consist of seven volumes with a total of more than 4100 pages and not all respondents have read the seven series, the text portion used for each respondent is different but similar. The text referenced will contain the following:

a. Harry Potter's actions that break the rules

b. heroic actions by Harry Potter or other figures

c. the brutality described by Voldemort or his accomplices

d. the use of spells to achieve a goal

The text is not for the respondents to re-read. It is referred to during the interview to remind them of the segment. It is to find out how far the segment has left an impression in the mind of the respondent and affect the respondent's affective. 
2. Interview.

Interviews with the respondents are conducted naively and in-depth directly based on the interview response. The following set of questions serves as a guideline for the main points. However, it does not rule out the possibility of adaptation of questions per the understanding of respondents and the addition of supporting questions to pursue responses given by respondents. The questions asked will be divided into several types:

a. Background questions, asking the respondent's data.

b. Affective questions, asking the respondent's affective responses to the Harry Potter texts, to see the extent to which the texts influence the respondent's value orientation.

c. Cognitive questions, asking the respondent's cognitive responses to the Harry Potter texts. These questions are to find out the respondent's critical thinking skills in distinguishing between fiction and fact. Besides, the questions examine the respondent's reasoning skills to grasp the values that the Harry Potter texts may contain.

The response to the interview questions is classified into three parts to analyze whether the respondent shows: an ability to distinguish between fiction and fact, the tendency to sympathize with occultism and violence as a result of reading Harry Potter texts, and an ability to identify the moral values in the texts.

\section{Research procedure}

This descriptive qualitative study uses narrative interpretation to describe its findings.

The materials analyzed are field notes, personal notes, and theory notes.

The steps of the study are as follows:

1. Record prospective respondents by conducting an initial interview.

2. Select five respondents through purposive sampling.

3. Select some books from the Harry Potter series and determine the portion of the book to be used as a research text.

4. Interview five respondents separately to find respondents' interpretations of the text.

5. Describe the results of the study based on direct observation and interviews with respondents.

The procedures for interpreting research data are as follows:

1. Classify the data into four major sections, namely: data about the background of the respondents, data that shows the ability of respondents to distinguish between fiction and fact, data that indicates the tendency of respondents to violence and occultism, and finally the data about respondents' learning of moral values on J.K. Rowling's Harry Potter texts.

2. Prepare a descriptive analysis of each respondent's background by taking into account the age of the respondent and comparing it with the stages of Piaget's cognitive development.

3. Compile a descriptive analysis of the respondent's background related to the level of religiosity in the family, including value education in the family, and the active role of parents in this value education. Considering that all respondents are from the same educational institution, it is assumed that all respondents received the same level of education on Christian values at school. In other words, value/religion education at school is treated as a constant, while value/religion education in the family as a variable that distinguishes one respondent from the others.

4. Prepare a descriptive analysis of the presence or absence of book discussion forums in the respondents' family and school. It is to examine how respondents overcome 
challenges they may face in understanding the Harry Potter texts, with whom respondents share the excitement about what they find in the text, as well as who influences respondents' understanding of the text.

5. Take note of any other additional information about the respondents found in the interview.

6. Conduct a descriptive analysis by referring to the evidence that shows the ability of respondents to make a distinction between fantasy (fiction) and reality (fact) by using Harry Potter texts as research instruments.

7. Analyze any evidence that leads to violence and occultism. The main points to examine here are the respondents' response to spells aimed at hurting others, the respondents' statement whether they like matters related to violence, as well as the occult in the text. Besides, the respondents' perceptions of occult symbols in the Harry Potter texts and the respondents' access to websites related to Harry Potter and Wicca as well as the extent to which respondents use such access. Observation of the respondents' daily behavior is also taken into consideration.

8. Conduct a descriptive analysis of the respondents' moral value learned from the Harry Potter texts. The main points observed here are the respondents' statements regarding the contents of the Harry Potter texts in general, their statements about what makes somebody a hero, and the characters or figures in the texts that respondents consider to be heroes. Then, the analysis is on the respondents' opinions on the values they can learn from the characters in the Harry Potter texts. Not all characters in the text are discussed; only characters who take an essential role in the whole story are discussed.

9. From all the data, a summary of respondents'profiles is compiled to see the extent to which the Harry Potter texts influence respondents in distinguishing between fiction and fact, causing respondents to become violent or sympathetic to the occult, and the extent to which the Harry Potter texts inspire moral/noble values to respondents.

\section{RESULTS AND DISCUSSION}

The table below shows a summary of the data analysis.

Table 1. Data analysis summary

\begin{tabular}{|c|c|c|c|c|c|}
\hline & $\mathbf{R} 1$ & R2 & R3 & R4 & R5 \\
\hline \multicolumn{6}{|l|}{ Personal data } \\
\hline Age & 13 & 13 & 13 & 11 & 12 \\
\hline Gender & female & female & Male & female & male \\
\hline Nationality & Filipino & Korean & Korean & Indonesian & Indonesian \\
\hline Grade/Year & 7 & 6 & 6 & 6 & 8 \\
\hline \multicolumn{6}{|c|}{ Family background } \\
\hline $\begin{array}{l}\text { Person provides } \\
\text { in the family }\end{array}$ & Father & Father & Father & Father & Father \\
\hline Family library & Yes & Yes & Yes & Yes & Yes \\
\hline $\begin{array}{l}\text { Democracy in } \\
\text { book choices }\end{array}$ & Yes & Yes & No & Yes & Yes \\
\hline $\begin{array}{l}\text { Parental guide in } \\
\text { book selection }\end{array}$ & Yes & No & $\begin{array}{l}\text { Yes, by } \\
\text { father }\end{array}$ & Yes & Yes \\
\hline $\begin{array}{l}\text { Discussion in } \\
\text { the family }\end{array}$ & $\begin{array}{l}\text { Yes, with } \\
\text { parents and } \\
\text { all family }\end{array}$ & $\begin{array}{l}\text { Almost none, } \\
\text { sometimes } \\
\text { with an older }\end{array}$ & No & $\begin{array}{c}\text { Yes, } \\
\text { supported by } \\
\text { both parents. }\end{array}$ & $\begin{array}{l}\text { Yes, } \\
\text { supported by } \\
\text { both parents. }\end{array}$ \\
\hline
\end{tabular}




\begin{tabular}{|c|c|c|c|c|c|}
\hline & R1 & $\mathbf{R 2}$ & $\mathbf{R 3}$ & $\mathbf{R 4}$ & R5 \\
\hline & members & $\begin{array}{l}\text { sibling } \\
\text { (sister) but } \\
\text { more about } \\
\text { general } \\
\text { matters, not } \\
\text { the book } \\
\text { content. }\end{array}$ & & & \\
\hline \multicolumn{6}{|c|}{ Religious background } \\
\hline Religion & $\begin{array}{l}\text { Catholic, } \\
\text { strong }\end{array}$ & $\begin{array}{l}\text { Christian, } \\
\text { weak }\end{array}$ & $\begin{array}{l}\text { Christian, } \\
\text { mediocre }\end{array}$ & $\begin{array}{l}\text { Christian, } \\
\text { strong }\end{array}$ & $\begin{array}{l}\text { Christian, } \\
\text { strong }\end{array}$ \\
\hline $\begin{array}{l}\text { Knowledge } \\
\text { about beliefs and } \\
\text { values in his/her } \\
\text { religion }\end{array}$ & $\begin{array}{l}\text { Must do } \\
\text { good deeds, } \\
\text { bad deeds } \\
\text { cause sin }\end{array}$ & No response & $\begin{array}{l}\text { Understands } \\
\text { the need to } \\
\text { read the } \\
\text { bible and } \\
\text { pray every } \\
\text { day to grow } \\
\text { as a } \\
\text { Christian }\end{array}$ & $\begin{array}{l}\text { Must grow } \\
\text { faith to God, } \\
\text { do not steal, } \\
\text { do not lie, do } \\
\text { not murder }\end{array}$ & $\begin{array}{l}\text { Faith and } \\
\text { characteris } \\
\text { important in } \\
\text { a religion }\end{array}$ \\
\hline $\begin{array}{l}\text { Knowledge } \\
\text { about what the } \\
\text { respondent's } \\
\text { religion restricts } \\
\text { in terms of } \\
\text { reading texts }\end{array}$ & No response & $\begin{array}{l}\text { Does not } \\
\text { know }\end{array}$ & Nothing & $\begin{array}{l}\text { Not allowed } \\
\text { to read other } \\
\text { religions' } \\
\text { bibles. }\end{array}$ & $\begin{array}{l}\text { Knows about } \\
\text { parts of the } \\
\text { Christian } \\
\text { scriptures } \\
\text { about good } \\
\text { reading texts } \\
\text { and those } \\
\text { forbidden by } \\
\text { the } \\
\text { scriptures, } \\
\text { including } \\
\text { texts related } \\
\text { to Wicca. }\end{array}$ \\
\hline $\begin{array}{l}\text { Knowledge of } \\
\text { the position of } \\
\text { the respondent's } \\
\text { religion in the } \\
\text { magic world and } \\
\text { Wicca }\end{array}$ & $\begin{array}{l}\text { Black magic } \\
\text { is forbidden } \\
\text { by religion. }\end{array}$ & No response & $\begin{array}{l}\text { Ghosts exist, } \\
\text { people who } \\
\text { practice } \\
\text { magic will } \\
\text { go to hell. }\end{array}$ & $\begin{array}{l}\text { Magic is the } \\
\text { power of } \\
\text { Satan. }\end{array}$ & $\begin{array}{l}\text { Magic is the } \\
\text { practice of } \\
\text { Satan. }\end{array}$ \\
\hline \multicolumn{6}{|l|}{ School background } \\
\hline $\begin{array}{l}\text { Book discussion } \\
\text { in the class }\end{array}$ & $\begin{array}{l}\text { Yes, but it } \\
\text { does not } \\
\text { discuss } \\
\text { books such } \\
\text { as Harry } \\
\text { Potter books. }\end{array}$ & No. & $\begin{array}{l}\text { Sometimes, } \\
\text { but it does } \\
\text { not discuss } \\
\text { texts like } \\
\text { Harry Potter } \\
\text { texts. }\end{array}$ & $\begin{array}{l}\text { Yes, but it } \\
\text { discusses } \\
\text { books related } \\
\text { to topics of } \\
\text { the study. }\end{array}$ & $\begin{array}{l}\text { Yes, it opens } \\
\text { to discussion } \\
\text { about any } \\
\text { book } \\
\text { including } \\
\text { Harry Potter } \\
\text { books. }\end{array}$ \\
\hline $\begin{array}{l}\text { Types of reading } \\
\text { texts discussed } \\
\text { in class }\end{array}$ & $\begin{array}{l}\text { Classic } \\
\text { stories }\end{array}$ & - & $\begin{array}{l}\text { Classic } \\
\text { stories }\end{array}$ & $\begin{array}{l}\text { Non-fiction } \\
\text { books related } \\
\text { to class } \\
\text { study. }\end{array}$ & $\begin{array}{l}\text { Bible, classic } \\
\text { stories }\end{array}$ \\
\hline $\begin{array}{l}\text { Methods of book } \\
\text { discussion in }\end{array}$ & $\begin{array}{l}\text { Presentations } \\
\text { and class }\end{array}$ & - & $\begin{array}{l}\text { Class } \\
\text { discussion }\end{array}$ & $\begin{array}{l}\text { Class } \\
\text { discussion }\end{array}$ & $\begin{array}{l}\text { Class } \\
\text { discussion }\end{array}$ \\
\hline
\end{tabular}




\begin{tabular}{|c|c|c|c|c|c|}
\hline & $\mathbf{R} 1$ & $\mathbf{R 2}$ & R3 & $\mathbf{R 4}$ & R5 \\
\hline class & discussions & & & & \\
\hline \multicolumn{6}{|l|}{ Book selection } \\
\hline $\begin{array}{l}\text { Reading } \\
\text { frequency }\end{array}$ & $\begin{array}{l}\text { Daily, 15-30 } \\
\text { minutes }\end{array}$ & $\begin{array}{l}\text { Almost } \\
\text { every day }\end{array}$ & $\begin{array}{l}\text { Every day, } \\
\text { around } 2 \\
\text { hours }\end{array}$ & $\begin{array}{l}\text { Every day, } \\
15-20 \\
\text { minutes }\end{array}$ & $\begin{array}{l}\text { Every day, } \\
\text { depending } \\
\text { on the needs }\end{array}$ \\
\hline Favorite genres & $\begin{array}{l}\text { Fiction, non- } \\
\text { fiction } \\
\text { (Times } \\
\text { magazines, } \\
\text { news about } \\
\text { celebrities) }\end{array}$ & Fiction & $\begin{array}{l}\text { Fiction and } \\
\text { non-fiction }\end{array}$ & Fiction & $\begin{array}{l}\text { Fiction about } \\
\text { fantasy and } \\
\text { horror, non- } \\
\text { fiction }\end{array}$ \\
\hline $\begin{array}{l}\text { Number of } \\
\text { Harry Potter } \\
\text { books read and } \\
\text { time spent on } \\
\text { the texts }\end{array}$ & $\begin{array}{l}7 \text { volumes in } \\
\text { English, } \\
\text { started } \\
\text { reading in } \\
\text { grade } 4\end{array}$ & $\begin{array}{l}6 \text { volumes in } \\
\text { Korean } \\
\text { language, } \\
\text { started } \\
\text { reading in } \\
\text { grade } 4\end{array}$ & $\begin{array}{l}5 \text { volumes - } \\
4 \text { volumes } \\
\text { Korean } \\
\text { language dan } \\
1 \text { in English, } \\
\text { started } \\
\text { reading in } \\
\text { grade } 4 .\end{array}$ & $\begin{array}{l}5 \text { volumes in } \\
\text { English, } \\
\text { started } \\
\text { reading in } \\
\text { grade } 5 \text {. Not } \\
\text { interested in } \\
\text { reading } \\
\text { volume } 6 \\
\text { and } 7 \text { yet. }\end{array}$ & $\begin{array}{l}7 \text { volumes in } \\
\text { English, } \\
\text { started to } \\
\text { read in grade } \\
4 .\end{array}$ \\
\hline $\begin{array}{l}\text { Initiatives/recom } \\
\text { mendations for } \\
\text { reading Harry } \\
\text { Potter texts }\end{array}$ & Father & Elder sister & $\begin{array}{l}\text { Own } \\
\text { initiative }\end{array}$ & $\begin{array}{l}\text { Elder } \\
\text { brother, } \\
\text { parents }\end{array}$ & $\begin{array}{l}\text { Own } \\
\text { initiative }\end{array}$ \\
\hline $\begin{array}{l}\text { Reasons to read } \\
\text { Harry Potter } \\
\text { texts }\end{array}$ & $\begin{array}{l}\text { Interesting } \\
\text { books }\end{array}$ & $\begin{array}{l}\text { Interested } \\
\text { because his } \\
\text { elder sister } \\
\text { read the } \\
\text { books }\end{array}$ & $\begin{array}{l}\text { Need } \\
\text { something to } \\
\text { read }\end{array}$ & $\begin{array}{l}\text { Parents' } \\
\text { recommenda } \\
\text { tion }\end{array}$ & curious \\
\hline \multicolumn{6}{|l|}{ Affective response } \\
\hline $\begin{array}{l}\text { Interpretation of } \\
\text { story content }\end{array}$ & $\begin{array}{l}\text { Harry Potter } \\
\text { survives } \\
\text { against } \\
\text { Voldemort, } \\
\text { who wants to } \\
\text { destroy him. }\end{array}$ & $\begin{array}{l}\text { Imagination, } \\
\text { magic, } \\
\text { adventure }\end{array}$ & $\begin{array}{l}\text { Adventure, } \\
\text { magic }\end{array}$ & $\begin{array}{l}\text { Adventure, } \\
\text { magic, } \\
\text { friendship }\end{array}$ & $\begin{array}{l}\text { The } \\
\text { adventure of } \\
\text { a boy with a } \\
\text { magic power } \\
\text { to fight } \\
\text { Voldemort } \\
\text { who } \\
\text { symbolizes } \\
\text { the power of } \\
\text { evil. }\end{array}$ \\
\hline $\begin{array}{l}\text { Feelings while } \\
\text { reading Harry } \\
\text { Potter }\end{array}$ & $\begin{array}{l}\text { Feels like he } \\
\text { were in the } \\
\text { book }\end{array}$ & $\begin{array}{l}\text { Sad, angry, } \\
\text { happy, } \\
\text { curious }\end{array}$ & $\begin{array}{l}\text { Fun, wants } \\
\text { to know } \\
\text { what will } \\
\text { happen later. }\end{array}$ & $\begin{array}{l}\text { Confused } \\
\text { because of } \\
\text { the British } \\
\text { English } \\
\text { terms used, } \\
\text { happy, sad, } \\
\text { nervous, } \\
\text { angry. }\end{array}$ & $\begin{array}{l}\text { Wants to } \\
\text { know what } \\
\text { happens } \\
\text { next. }\end{array}$ \\
\hline $\begin{array}{l}\text { Favorite parts of } \\
\text { the Harry Potter } \\
\text { texts }\end{array}$ & $\begin{array}{l}\text { Triwizard } \\
\text { Tournament, } \\
\text { when Harry }\end{array}$ & $\begin{array}{l}\text { When Harry } \\
\text { fought off } \\
\text { the basilisk }\end{array}$ & $\begin{array}{l}\text { Magic, } \\
\text { magical } \\
\text { creatures, }\end{array}$ & $\begin{array}{l}\text { Food } \\
\text { descriptions, } \\
\text { riding a }\end{array}$ & $\begin{array}{l}\text { In general: } \\
\text { language } \\
\text { style, }\end{array}$ \\
\hline
\end{tabular}




\begin{tabular}{|c|c|c|c|c|c|}
\hline & R1 & $\mathbf{R 2}$ & R3 & $\mathbf{R 4}$ & R5 \\
\hline & $\begin{array}{l}\text { dived to save } \\
\text { the captives }\end{array}$ & $\begin{array}{l}\text { (a giant } \\
\text { snake), } \\
\text { Harry was } \\
\text { injured and } \\
\text { cured with } \\
\text { phoenix } \\
\text { tears. } \\
\text { Finally, } \\
\text { Harry could } \\
\text { kill the } \\
\text { basilisk. }\end{array}$ & $\begin{array}{l}\text { Hagrid (one } \\
\text { of the } \\
\text { characters in } \\
\text { the text). }\end{array}$ & $\begin{array}{l}\text { broomstick, } \\
\text { isits to the } \\
\text { candy shop } \\
\text { in } \\
\text { Hogsmeade. }\end{array}$ & $\begin{array}{l}\text { creativity, } \\
\text { character, } \\
\text { and romance. } \\
\text { Specifically: } \\
\text { quidditch } \\
\text { matches, } \\
\text { Harry's battle } \\
\text { with } \\
\text { Voldemort, } \\
\text { children } \\
\text { petrified by } \\
\text { the basilisk's } \\
\text { black magic, } \\
\text { the candy } \\
\text { shop at } \\
\text { Hogsmeade, } \\
\text { Triwizard } \\
\text { competitions } \\
\text {, romantic } \\
\text { parts. }\end{array}$ \\
\hline $\begin{array}{l}\text { Unpleasant parts } \\
\text { of Harry Potter } \\
\text { texts }\end{array}$ & $\begin{array}{l}\text { Voldemort's } \\
\text { revival } \\
\text { ceremony, } \\
\text { Harry's love } \\
\text { story } \\
\text { (because it } \\
\text { doesn't fit the } \\
\text { mystery } \\
\text { theme of the } \\
\text { text) }\end{array}$ & $\begin{array}{l}\text { When Harry } \\
\text { was bitten by } \\
\text { the basilisk; } \\
\text { inferi troops } \\
\text { (zombies), } \\
\text { fighting } \\
\text { scenes and } \\
\text { the sad parts }\end{array}$ & None. & $\begin{array}{l}\text { Scary parts, } \\
\text { such as the } \\
\text { battle } \\
\text { between } \\
\text { Harry and } \\
\text { the basilisk, } \\
\text { dementors; } \\
\text { the fight } \\
\text { between } \\
\text { Harry and } \\
\text { Malfoy, } \\
\text { accusations } \\
\text { against } \\
\text { Harry. }\end{array}$ & $\begin{array}{l}\text { Difficult } \\
\text { words, harsh } \\
\text { words (e.g., } \\
\text { bloody hell), } \\
\text { whiny parts } \\
\text { (e.g., } \\
\text { Hermione } \\
\text { who cries a } \\
\text { lot) }\end{array}$ \\
\hline \multicolumn{6}{|c|}{ Cognitive response } \\
\hline $\begin{array}{l}\text { Opportunity for } \\
\text { discussion about } \\
\text { Harry Potter } \\
\text { texts/ discussion } \\
\text { partners }\end{array}$ & $\begin{array}{l}\text { Yes, with } \\
\text { father and } \\
\text { two elder } \\
\text { sisters. }\end{array}$ & $\begin{array}{l}\text { Yes, with an } \\
\text { elder sister; } \\
\text { discussion is } \\
\text { more about } \\
\text { clarifying } \\
\text { what } \\
\text { happens in } \\
\text { the story, not } \\
\text { about the } \\
\text { content. }\end{array}$ & No. & $\begin{array}{l}\text { Yes, with an } \\
\text { elder brother. }\end{array}$ & $\begin{array}{l}\text { Yes, with } \\
\text { fellow Harry } \\
\text { Potter fans, } \\
\text { with family. }\end{array}$ \\
\hline $\begin{array}{l}\text { The ability to } \\
\text { distinguish } \\
\text { between fiction } \\
\text { and fact }\end{array}$ & $\begin{array}{l}\text { Demonstrate } \\
\text { s an ability } \\
\text { to } \\
\text { distinguish } \\
\text { between } \\
\text { fiction and }\end{array}$ & $\begin{array}{l}\text { Demonstrate } \\
\text { s an ability } \\
\text { to } \\
\text { distinguish } \\
\text { between } \\
\text { fiction and }\end{array}$ & $\begin{array}{l}\text { Demonstrate } \\
\text { s an ability } \\
\text { to } \\
\text { distinguish } \\
\text { between } \\
\text { fiction and }\end{array}$ & $\begin{array}{l}\text { Demonstrate } \\
\text { s an ability } \\
\text { to } \\
\text { distinguish } \\
\text { between } \\
\text { fiction and }\end{array}$ & $\begin{array}{l}\text { Demonstrate } \\
\text { s an ability } \\
\text { to } \\
\text { distinguish } \\
\text { between } \\
\text { fictionand }\end{array}$ \\
\hline
\end{tabular}




\begin{tabular}{|c|c|c|c|c|c|}
\hline & R1 & $\mathbf{R 2}$ & $\mathbf{R 3}$ & $\mathbf{R 4}$ & R5 \\
\hline & fact & fact & fact & fact & fact \\
\hline $\begin{array}{l}\text { Harry Potter's } \\
\text { eligibility for a } \\
\text { school library }\end{array}$ & $\begin{array}{l}\text { Yes, Harry } \\
\text { Potter texts } \\
\text { do not } \\
\text { expose } \\
\text { violence. }\end{array}$ & $\begin{array}{l}\text { Yes, because } \\
\text { such an } \\
\text { English- } \\
\text { language } \\
\text { novel can } \\
\text { provide a } \\
\text { complete } \\
\text { reading. }\end{array}$ & $\begin{array}{l}\text { Yes, because } \\
\text { many people } \\
\text { want to read } \\
\text { the book. }\end{array}$ & $\begin{array}{l}\text { Yes, so } \\
\text { people know } \\
\text { that Harry } \\
\text { Potter books } \\
\text { aren't boring. }\end{array}$ & $\begin{array}{l}\text { Yes, but for } \\
\text { readers of a } \\
\text { certain age. }\end{array}$ \\
\hline $\begin{array}{l}\text { The influence of } \\
\text { Harry Potter on } \\
\text { learning } \\
\text { achievement }\end{array}$ & $\begin{array}{l}\text { Yes, there is } \\
\text { an increase } \\
\text { in thinking } \\
\text { skills } \\
\text { specifically } \\
\text { in predicting }\end{array}$ & None. & $\begin{array}{l}\text { Yes, there is } \\
\text { an } \\
\text { improvement } \\
\text { in the ability } \\
\text { to read and } \\
\text { write in } \\
\text { Korean } \\
\text { language, } \\
\text { adding } \\
\text { vocabulary } \\
\text { in English. }\end{array}$ & None. & $\begin{array}{l}\text { Yes, there is } \\
\text { an increase } \\
\text { in English } \\
\text { language } \\
\text { skills. }\end{array}$ \\
\hline \multicolumn{6}{|l|}{ Magic and spells } \\
\hline $\begin{array}{l}\text { Definition of } \\
\text { magic }\end{array}$ & $\begin{array}{l}\text { Spells that } \\
\text { help people }\end{array}$ & $\begin{array}{l}\text { A fantasy. } \\
\text { The magic in } \\
\text { Harry Potter } \\
\text { is only an } \\
\text { illusion, but } \\
\text { magic tricks } \\
\text { such as in } \\
\text { card magic } \\
\text { tricks are } \\
\text { real. }\end{array}$ & $\begin{array}{l}\text { Strange } \\
\text { actions that } \\
\text { can surprise } \\
\text { people and } \\
\text { make people } \\
\text { famous. }\end{array}$ & $\begin{array}{l}\text { Just a } \\
\text { fantasy. }\end{array}$ & $\begin{array}{l}\text { A } \\
\text { supernatural } \\
\text { power } \\
\text { resulting } \\
\text { from a } \\
\text { combination } \\
\text { of specific } \\
\text { movements } \\
\text { and certain } \\
\text { spells. }\end{array}$ \\
\hline $\begin{array}{l}\text { The existence of } \\
\text { magic in real life }\end{array}$ & No & No & $\begin{array}{l}\text { Yes, because } \\
\text { it was once } \\
\text { shown on } \\
\text { TV. }\end{array}$ & $\begin{array}{l}\text { No, if there } \\
\text { is, it must be } \\
\text { the practice } \\
\text { of demonic } \\
\text { power. }\end{array}$ & $\begin{array}{l}\text { Not sure, } \\
\text { maybe there } \\
\text { is because } \\
\text { based on } \\
\text { ancient } \\
\text { history there } \\
\text { were } \\
\text { magicians; } \\
\text { but even if } \\
\text { there are } \\
\text { wizards, } \\
\text { there aren't } \\
\text { many. }\end{array}$ \\
\hline $\begin{array}{l}\text { Definition of a } \\
\text { mantra }\end{array}$ & $\begin{array}{l}\text { Words with } \\
\text { magic power }\end{array}$ & $\begin{array}{l}\text { Words with } \\
\text { power so that } \\
\text { when spoken } \\
\text { they can } \\
\text { make } \\
\text { something } \\
\text { happen. }\end{array}$ & $\begin{array}{l}\text { Words used } \\
\text { by someone } \\
\text { who will use } \\
\text { magic } \\
\text { power. }\end{array}$ & $\begin{array}{l}\text { Words that } \\
\text { have the } \\
\text { power to } \\
\text { make things } \\
\text { happen. }\end{array}$ & $\begin{array}{l}\text { Words that } \\
\text { have the } \\
\text { power to } \\
\text { make things } \\
\text { happen. }\end{array}$ \\
\hline Try the Harry & Yes & Yes & Yes & No & Yes \\
\hline
\end{tabular}




\begin{tabular}{|c|c|c|c|c|c|}
\hline & R1 & $\mathbf{R} 2$ & R3 & R4 & R5 \\
\hline \multicolumn{6}{|l|}{ Potter spells } \\
\hline $\begin{array}{l}\text { Favorite spells } \\
\& \text { reason }\end{array}$ & $\begin{array}{l}\text { Reducto(soli } \\
\text { d object } \\
\text { explosive } \\
\text { spells) } \\
\text { because it's } \\
\text { cool }\end{array}$ & $\begin{array}{l}\text { Accio (spell } \\
\text { for calling } \\
\text { objects), } \\
\text { because the } \\
\text { respondent } \\
\text { does not } \\
\text { need to walk } \\
\text { to retrieve } \\
\text { the object he } \\
\text { needs. }\end{array}$ & All. & $\begin{array}{l}\text { Expecto } \\
\text { Patronum, } \\
\text { because it } \\
\text { can drive out } \\
\text { dementors. }\end{array}$ & $\begin{array}{l}\text { Imperio(spell } \\
\text { for } \\
\text { controllingpe } \\
\text { ople) and } \\
\text { Cruciatus } \\
\text { (spell for } \\
\text { torturing), } \\
\text { because they } \\
\text { can be used } \\
\text { to threaten } \\
\text { people to do } \\
\text { what they } \\
\text { want. } \\
\text { Carpero } \\
\text { Retractum(sp } \\
\text { ell calling } \\
\text { objects), } \\
\text { because the } \\
\text { respondent } \\
\text { does not } \\
\text { need to walk } \\
\text { to retrieve } \\
\text { the items } \\
\text { needed. }\end{array}$ \\
\hline $\begin{array}{l}\text { Least favorite } \\
\text { spell }\end{array}$ & $\begin{array}{l}\text { Wingardium } \\
\text { Leviosa(spell } \\
\text { for lifting } \\
\text { something), } \\
\text { because it's } \\
\text { too trivial. }\end{array}$ & $\begin{array}{l}\text { Sectum- } \\
\text { sempra(spell } \\
\text { s that slice } \\
\text { the victim), } \\
\text { because this } \\
\text { spell can hurt } \\
\text { people. }\end{array}$ & $\begin{array}{l}\text { Avada } \\
\text { Kedavra(killi } \\
\text { ng spell), } \\
\text { because it } \\
\text { can kill } \\
\text { people in an } \\
\text { instant. }\end{array}$ & $\begin{array}{l}\text { Avada } \\
\text { Kedavra, } \\
\text { because this } \\
\text { spell hurts } \\
\text { people. }\end{array}$ & $\begin{array}{l}\text { Avada } \\
\text { Kedavra } \\
\text { because this } \\
\text { spell is cruel. } \\
\text { Engorgio } \\
\text { (spell for } \\
\text { enlargingthe } \\
\text { size an } \\
\text { object), } \\
\text { Reducio } \\
\text { (spell for } \\
\text { reducing the } \\
\text { size of } \\
\text { anobject), } \\
\text { Incendio } \\
\text { (lighting a } \\
\text { fire), hair } \\
\text { straighten- } \\
\text { ing spell; } \\
\text { because the } \\
\text { spells are } \\
\text { trivial and } \\
\text { childish. }\end{array}$ \\
\hline Cruel spells & $\begin{array}{l}\text { Crucio\&Imp } \\
\text { erio } \\
\text { (torturing } \\
\text { spell) }\end{array}$ & $\begin{array}{l}\text { Sectumsem- } \\
\text { pra(slicing } \\
\text { spell) }\end{array}$ & $\begin{array}{l}\text { Avada } \\
\text { Kedavra(killi } \\
\text { ng spell) }\end{array}$ & $\begin{array}{l}\text { Avada } \\
\text { Kedavra(killi } \\
\text { ng spell) }\end{array}$ & $\begin{array}{l}\text { Avada } \\
\text { Kedavra(killi } \\
\text { ng spell) }\end{array}$ \\
\hline
\end{tabular}




\begin{tabular}{|c|c|c|c|c|c|}
\hline & R1 & $\mathbf{R 2}$ & R3 & R4 & R5 \\
\hline \multicolumn{6}{|l|}{ Magic World } \\
\hline $\begin{array}{l}\text { Existence of } \\
\text { ghost }\end{array}$ & $\begin{array}{l}\text { Ghosts do } \\
\text { not exist, but } \\
\text { dementors } \\
\text { exist. }\end{array}$ & $\begin{array}{l}\text { Ghosts may } \\
\text { exist, not } \\
\text { sure. }\end{array}$ & $\begin{array}{l}\text { Ghosts exist. } \\
\text { (The } \\
\text { respondent } \\
\text { claims that } \\
\text { his religion } \\
\text { acknowledge } \\
\text { s the } \\
\text { existence of } \\
\text { ghosts) }\end{array}$ & $\begin{array}{l}\text { Ghosts do } \\
\text { not exist. }\end{array}$ & $\begin{array}{l}\text { Ghosts do } \\
\text { not exist. }\end{array}$ \\
\hline $\begin{array}{l}\text { Reason } \\
\text { whythere are } \\
\text { ghosts in the } \\
\text { Harry Potter } \\
\text { texts }\end{array}$ & $\begin{array}{l}\text { Doesn't } \\
\text { know }\end{array}$ & $\begin{array}{l}\text { Fantasy, to } \\
\text { make the } \\
\text { story more } \\
\text { interesting }\end{array}$ & $\begin{array}{l}\text { To make the } \\
\text { story more } \\
\text { interesting }\end{array}$ & $\begin{array}{l}\text { To make the } \\
\text { story more } \\
\text { interesting }\end{array}$ & $\begin{array}{l}\text { To add an } \\
\text { element of } \\
\text { humor to the } \\
\text { story and } \\
\text { make people } \\
\text { not afraid of } \\
\text { ghosts } \\
\text { because } \\
\text { ghosts in the } \\
\text { Harry Potter } \\
\text { texts are } \\
\text { funny. }\end{array}$ \\
\hline \multicolumn{6}{|c|}{ Animals \& animagus } \\
\hline $\begin{array}{l}\text { Special animals } \\
\text { in Harry Potter }\end{array}$ & $\begin{array}{l}\text { Hippogriff } \\
\text { (magical } \\
\text { animal that is } \\
\text { a } \\
\text { combination } \\
\text { between a } \\
\text { winged horse } \\
\text { and an } \\
\text { eagle's head) }\end{array}$ & Hippogriff & $\begin{array}{l}\text { Griffin } \\
\text { (Greek } \\
\text { mythical } \\
\text { animal with } \\
\text { a giant } \\
\text { hawk's head } \\
\text { and front } \\
\text { legs, a lion's } \\
\text { body and } \\
\text { hind legs) }\end{array}$ & $\begin{array}{l}\text { Hippogriff, } \\
\text { owl, and } \\
\text { thestral. }\end{array}$ & $\begin{array}{l}\text { Hippogriff, } \\
\text { thestral, } \\
\text { gygmy puff } \\
\text { (animals } \\
\text { shaped like } \\
\text { hair balls, } \\
\text { brightly } \\
\text { colored) }\end{array}$ \\
\hline $\begin{array}{l}\text { Possible } \\
\text { existence of } \\
\text { animagus in the } \\
\text { real world }\end{array}$ & $\begin{array}{l}\text { Impossible, } \\
\text { no one } \\
\text { would want } \\
\text { to be an } \\
\text { animal }\end{array}$ & Impossible & $\begin{array}{l}\text { Impossible, } \\
\text { because it's } \\
\text { too } \\
\text { dangerous to } \\
\text { be an animal. }\end{array}$ & Impossible & Impossible \\
\hline $\begin{array}{l}\text { Knowledge of } \\
\text { animals in } \\
\text { legends and } \\
\text { mythology }\end{array}$ & $\begin{array}{l}\text { Good, knows } \\
\text { abouttembala } \\
\text { ngwhich is a } \\
\text { kind of } \\
\text { centaurs that } \\
\text { exist in } \\
\text { Philippine } \\
\text { legend. }\end{array}$ & $\begin{array}{l}\text { Does not } \\
\text { know } \\
\text { animals in } \\
\text { legends and } \\
\text { mythology } \\
\text { before } \\
\text { reading } \\
\text { Harry Potter } \\
\text { texts }\end{array}$ & $\begin{array}{l}\text { Good, gets to } \\
\text { know } \\
\text { centaurs } \\
\text { from books } \\
\text { on } \\
\text { mythology }\end{array}$ & $\begin{array}{l}\text { Good, gets to } \\
\text { know the } \\
\text { centaursfrom } \\
\text { the book of } \\
\text { Narnia }\end{array}$ & $\begin{array}{l}\text { Gets to know } \\
\text { centaursat } \\
\text { the first time } \\
\text { from the } \\
\text { Harry Potter } \\
\text { texts }\end{array}$ \\
\hline \multicolumn{6}{|l|}{ History } \\
\hline $\begin{array}{l}\text { Influence of } \\
\text { knowledge of } \\
\text { historical } \\
\text { background on }\end{array}$ & No influence & $\begin{array}{l}\text { No } \\
\text { knowledge } \\
\text { of historical } \\
\text { background }\end{array}$ & $\begin{array}{l}\text { No } \\
\text { knowledge } \\
\text { of relevant } \\
\text { historical }\end{array}$ & $\begin{array}{l}\text { No } \\
\text { knowledge } \\
\text { of relevant } \\
\text { historical }\end{array}$ & $\begin{array}{l}\text { Not sure } \\
\text { about the } \\
\text { presence or } \\
\text { absence of }\end{array}$ \\
\hline
\end{tabular}




\begin{tabular}{|c|c|c|c|c|c|}
\hline & R1 & $\mathbf{R} 2$ & $\mathbf{R 3}$ & R4 & R5 \\
\hline $\begin{array}{l}\text { the ability to } \\
\text { identify fiction } \\
\text { and facts }\end{array}$ & & & background & background & $\begin{array}{l}\text { witches due } \\
\text { to historical } \\
\text { facts about } \\
\text { the slaughter } \\
\text { of witches in } \\
\text { the 18th } \\
\text { century }\end{array}$ \\
\hline $\begin{array}{l}\text { Opinions on } \\
\text { historical facts } \\
\text { about the } \\
\text { slaughter of } \\
\text { witches }\end{array}$ & $\begin{array}{l}\text { Doesn't } \\
\text { know the } \\
\text { historical } \\
\text { facts about } \\
\text { the slaughter } \\
\text { of witches. } \\
\text { Magicians } \\
\text { are human so } \\
\text { they must be } \\
\text { treated like } \\
\text { other } \\
\text { humans. }\end{array}$ & $\begin{array}{l}\text { Doesn't } \\
\text { know the } \\
\text { historical } \\
\text { facts about } \\
\text { the slaughter } \\
\text { of witches. } \\
\text { Bad witches } \\
\text { should be } \\
\text { killed. Bad } \\
\text { and good } \\
\text { witches can } \\
\text { be } \\
\text { distinguished } \\
\text { from what } \\
\text { they do. }\end{array}$ & $\begin{array}{l}\text { Doesn't } \\
\text { know the } \\
\text { historical } \\
\text { facts about } \\
\text { the slaughter } \\
\text { of witches. } \\
\text { Bad witches } \\
\text { should be } \\
\text { killed. Bad } \\
\text { and good } \\
\text { witches can } \\
\text { be } \\
\text { distinguished } \\
\text { from what } \\
\text { they do. }\end{array}$ & $\begin{array}{l}\text { Doesn't } \\
\text { know the } \\
\text { historical } \\
\text { facts about } \\
\text { the slaughter } \\
\text { of witches. } \\
\text { Evil witches } \\
\text { should not be } \\
\text { allowed to } \\
\text { live freely } \\
\text { because they } \\
\text { can endanger } \\
\text { others. }\end{array}$ & $\begin{array}{l}\text { Knows the } \\
\text { historical } \\
\text { facts about } \\
\text { the slaughter } \\
\text { of witches, } \\
\text { agrees that } \\
\text { evil witches } \\
\text { should be } \\
\text { eliminated } \\
\text { by arrest, } \\
\text { trial and } \\
\text { imprisonmen } \\
\text { t. }\end{array}$ \\
\hline \multicolumn{6}{|c|}{ Fortune telling\& fortune tellers } \\
\hline $\begin{array}{l}\text { Opinions about } \\
\text { fortune telling }\end{array}$ & Sheer lies. & Sheer lies. & $\begin{array}{l}\text { Only part of } \\
\text { it is true. }\end{array}$ & Sheer lies. & Sheer lies. \\
\hline $\begin{array}{l}\text { Opinions about } \\
\text { fortune tellers }\end{array}$ & $\begin{array}{l}\text { A fortune } \\
\text { teller might } \\
\text { really be } \\
\text { there or just } \\
\text { someone } \\
\text { who is } \\
\text { insane }\end{array}$ & $\begin{array}{l}\text { A fortune } \\
\text { teller is a } \\
\text { liar. }\end{array}$ & $\begin{array}{l}\text { Some are } \\
\text { just lying, } \\
\text { some are } \\
\text { telling the } \\
\text { truth. }\end{array}$ & $\begin{array}{l}\text { A fortune } \\
\text { teller is a con } \\
\text { artist. }\end{array}$ & $\begin{array}{l}\text { There is no } \\
\text { way anyone } \\
\text { can say what } \\
\text { will happen } \\
\text { in the future. }\end{array}$ \\
\hline \multicolumn{6}{|l|}{ Occultism } \\
\hline $\begin{array}{l}\text { Definition of } \\
\text { crime }\end{array}$ & $\begin{array}{l}\text { Lying, doing } \\
\text { evil things, } \\
\text { and not } \\
\text { regretting it }\end{array}$ & $\begin{array}{l}\text { Something } \\
\text { bad }\end{array}$ & $\begin{array}{l}\text { Doing bad } \\
\text { things to } \\
\text { others }\end{array}$ & $\begin{array}{l}\text { Like to kill, } \\
\text { like to } \\
\text { disguise }\end{array}$ & $\begin{array}{l}\text { A } \\
\text { combination } \\
\text { of egoism, } \\
\text { greed, and } \\
\text { power }\end{array}$ \\
\hline $\begin{array}{l}\text { Able to } \\
\text { recognize occult } \\
\text { symbols on the } \\
\text { forehead of the } \\
\text { main character } \\
\text { Harry Potter }\end{array}$ & No & No & No & No & $\begin{array}{l}\text { No; does not } \\
\text { pay attention } \\
\text { to this } \\
\text { wound } \\
\text { because he is } \\
\text { more } \\
\text { focused on } \\
\text { the use of } \\
\text { language in } \\
\text { the texts. }\end{array}$ \\
\hline $\begin{array}{l}\text { Tendency to } \\
\text { occultism }\end{array}$ & Not found & Not found & Not found & Not found & Not found \\
\hline
\end{tabular}




\begin{tabular}{|c|c|c|c|c|c|}
\hline & R1 & R2 & R3 & R4 & R5 \\
\hline \multicolumn{6}{|l|}{ Heroism } \\
\hline $\begin{array}{l}\text { Definition of } \\
\text { heroism }\end{array}$ & $\begin{array}{l}\text { People who } \\
\text { are admired } \\
\text { for helping } \\
\text { others }\end{array}$ & $\begin{array}{l}\text { People who } \\
\text { dare to take } \\
\text { risks, save } \\
\text { the weak, } \\
\text { work hard, } \\
\text { try to be } \\
\text { good, wise }\end{array}$ & $\begin{array}{l}\text { People who } \\
\text { do good for } \\
\text { society }\end{array}$ & $\begin{array}{l}\text { Someone } \\
\text { who saves } \\
\text { others }\end{array}$ & $\begin{array}{l}\text { Someone } \\
\text { who saves } \\
\text { the lives of } \\
\text { others, who } \\
\text { helps others }\end{array}$ \\
\hline $\begin{array}{l}\text { The characters } \\
\text { of a hero }\end{array}$ & $\begin{array}{l}\text { patient, } \\
\text { gentle, } \\
\text { generous }\end{array}$ & $\begin{array}{l}\text { Risk taker, } \\
\text { helping the } \\
\text { weak, kind, } \\
\text { hard worker. }\end{array}$ & $\begin{array}{l}\text { Doing good } \\
\text { deeds }\end{array}$ & $\begin{array}{l}\text { Caring, } \\
\text { open- } \\
\text { minded, } \\
\text { knowledgeab } \\
\text { le, } \\
\text { responsible,t } \\
\text { hinker, } \\
\text { reflective. }\end{array}$ & $\begin{array}{l}\text { Humble, } \\
\text { unselfish, } \\
\text { kind, } \\
\text { someone } \\
\text { who is loved } \\
\text { by many } \\
\text { people, a } \\
\text { popular } \\
\text { person }\end{array}$ \\
\hline $\begin{array}{l}\text { Favorite } \\
\text { character in } \\
\text { Harry Potter \& } \\
\text { reason }\end{array}$ & $\begin{array}{l}\text { Ronald } \\
\text { Weasley } \\
\text { because this } \\
\text { character is } \\
\text { more } \\
\text { "humane" - } \\
\text { sometimes } \\
\text { evil, } \\
\text { sometimes } \\
\text { good, } \\
\text { sometimes } \\
\text { acting stupid, } \\
\text { sometimes } \\
\text { having a } \\
\text { brilliant idea. }\end{array}$ & $\begin{array}{l}\text { Harry, } \\
\text { Hermione, } \\
\text { Ron, Sirius } \\
\text { Black; } \\
\text { because } \\
\text { Hermione, } \\
\text { Ron, and } \\
\text { Sirius helped } \\
\text { Harry Potter. }\end{array}$ & $\begin{array}{l}\text { Harry Potter, } \\
\text { because } \\
\text { Harry is the } \\
\text { main } \\
\text { character in } \\
\text { the story, a } \\
\text { risk taker, he } \\
\text { has good } \\
\text { magic and } \\
\text { cares for his } \\
\text { friends }\end{array}$ & $\begin{array}{l}\text { Harry, } \\
\text { Hermione, } \\
\text { and Ron, } \\
\text { because they } \\
\text { deal with } \\
\text { dangerous } \\
\text { situations } \\
\text { and they are } \\
\text { always able } \\
\text { to overcome } \\
\text { them }\end{array}$ & $\begin{array}{l}\text { Harry, } \\
\text { Hermione, } \\
\text { and Ron. } \\
\text { Harry is very } \\
\text { brave, kind, } \\
\text { and } \\
\text { confident. } \\
\text { Ron has a } \\
\text { sense of } \\
\text { humor and } \\
\text { wants to get } \\
\text { things done } \\
\text { quickly. } \\
\text { Hermione is } \\
\text { very } \\
\text { intelligent, } \\
\text { kind, and } \\
\text { plays an } \\
\text { important } \\
\text { role in } \\
\text { Harry's } \\
\text { fights to } \\
\text { defeat } \\
\text { Voldemort. }\end{array}$ \\
\hline $\begin{array}{l}\text { The heroes in } \\
\text { the Harry Potter } \\
\text { text }\end{array}$ & $\begin{array}{l}\text { Harry, } \\
\text { Hermione, } \\
\text { Ron, Snape }\end{array}$ & $\begin{array}{l}\text { Harry, } \\
\text { Hermione, } \\
\text { Ron, Sirius } \\
\text { Black. }\end{array}$ & Dumbledore & $\begin{array}{l}\text { Harry, } \\
\text { Dumbledore, } \\
\text { Hagrid, } \\
\text { Snape, Sirius } \\
\text { Black, } \\
\text { Dobby, } \\
\text { Hermione, } \\
\text { Ron. }\end{array}$ & Harry Potter \\
\hline \multicolumn{6}{|c|}{ Distinction between fiction and fact } \\
\hline $\begin{array}{l}\text { The ability to } \\
\text { distinguish the }\end{array}$ & $\begin{array}{l}\text { Capable, } \\
\text { very rational }\end{array}$ & Capable & Capable & Capable & Capable \\
\hline
\end{tabular}




\begin{tabular}{|c|c|c|c|c|c|}
\hline & R1 & $\mathbf{R 2}$ & R3 & R4 & R5 \\
\hline \multicolumn{6}{|l|}{$\begin{array}{l}\text { world of Harry } \\
\text { Potter and the } \\
\text { real world of } \\
\text { respondents }\end{array}$} \\
\hline \multicolumn{6}{|c|}{ Harry Potter as an inspiration } \\
\hline $\begin{array}{l}\text { Respondent's } \\
\text { empathy for } \\
\text { Harry Potter's } \\
\text { characters }\end{array}$ & $\begin{array}{l}\text { Harry } \\
\text { sometimes } \\
\text { violates the } \\
\text { rules, but } \\
\text { there is a } \\
\text { noble } \\
\text { purpose } \\
\text { behind the } \\
\text { violation. }\end{array}$ & $\begin{array}{l}\text { Harry is not } \\
\text { perfect as a } \\
\text { hero, but no } \\
\text { one is perfect } \\
\text { in this world. }\end{array}$ & $\begin{array}{l}\text { Harry } \\
\text { sometimes } \\
\text { does the } \\
\text { wrong thing, } \\
\text { but basically } \\
\text { he is kind, he } \\
\text { is not afraid } \\
\text { of anything, } \\
\text { and cares for } \\
\text { his friends. }\end{array}$ & $\begin{array}{l}\text { Harry } \\
\text { sometimes } \\
\text { does things } \\
\text { that break } \\
\text { the rules, but } \\
\text { he has a } \\
\text { certain } \\
\text { reason, } \\
\text { which is for } \\
\text { good. So this } \\
\text { kind of } \\
\text { action can be } \\
\text { justified. }\end{array}$ & $\begin{array}{l}\text { Harry } \\
\text { sometimes } \\
\text { violates the } \\
\text { rules, but if } \\
\text { the goal is } \\
\text { good then it } \\
\text { can be } \\
\text { considered } \\
\text { right. }\end{array}$ \\
\hline $\begin{array}{l}\text { Harry Potter's } \\
\text { influence in } \\
\text { inspiring } \\
\text { problem solving }\end{array}$ & $\begin{array}{l}\text { Try as little } \\
\text { as possible to } \\
\text { bother } \\
\text { others, but if } \\
\text { the problem } \\
\text { is too big we } \\
\text { can ask for } \\
\text { help. } \\
\text { Friends can } \\
\text { help us solve } \\
\text { problems. }\end{array}$ & $\begin{array}{l}\text { Disputes } \\
\text { with friends } \\
\text { are common } \\
\text { among } \\
\text { friends. If } \\
\text { such disputes } \\
\text { occur, a } \\
\text { sincere } \\
\text { apology can } \\
\text { be a } \\
\text { powerful } \\
\text { way of } \\
\text { reconciliatio } \\
\text { n. }\end{array}$ & Nothing. & $\begin{array}{l}\text { Be a person } \\
\text { who is } \\
\text { caring, } \\
\text { brave, } \\
\text { independent, } \\
\text { and can } \\
\text { forgive the } \\
\text { mistakes of } \\
\text { others. Be } \\
\text { honest, don't } \\
\text { lie unless } \\
\text { you lie for a } \\
\text { good cause. }\end{array}$ & $\begin{array}{l}\text { If other } \\
\text { people don't } \\
\text { like us, then } \\
\text { we must do } \\
\text { good to them } \\
\text { so they like } \\
\text { us again. }\end{array}$ \\
\hline \multicolumn{6}{|l|}{ Consumerism } \\
\hline $\begin{array}{l}\text { Respondent's } \\
\text { collection of } \\
\text { Harry Potter } \\
\text { merchandise }\end{array}$ & A cup & A notebook & Nothing & Nothing & $\begin{array}{l}\text { clocks, } \\
\text { rulers, } \\
\text { watches, } \\
\text { posters, } \\
\text { books, } \\
\text { wallpapers, } \\
\text { drawings, } \\
\text { films, } \\
\text { pillows, } \\
\text { blankets, bed } \\
\text { linen, pencil } \\
\text { cases, } \\
\text { pencils, } \\
\text { sweaters, T- } \\
\text { shirts. }\end{array}$ \\
\hline $\begin{array}{l}\text { Harry } \\
\text { PotterGames }\end{array}$ & 1 & No & 1 & 1 & 1 \\
\hline $\begin{array}{l}\text { Harry Potter } \\
\text { books }\end{array}$ & $\begin{array}{l}7 \text { full } \\
\text { volumes }\end{array}$ & 6 volumes & 5 volumes & $\begin{array}{l}7 \text { full } \\
\text { volumes }\end{array}$ & $\begin{array}{l}7 \text { full } \\
\text { volumes }\end{array}$ \\
\hline
\end{tabular}




\begin{tabular}{|c|c|c|c|c|c|}
\hline & $\mathbf{R} 1$ & $\mathbf{R 2}$ & R3 & R4 & R5 \\
\hline $\begin{array}{l}\text { Possess other } \\
\text { books related to } \\
\text { Harry Potter }\end{array}$ & $\begin{array}{l}\text { No, but } \\
\text { wants to } \\
\text { have }\end{array}$ & No & No & No & $\begin{array}{l}\text { No, but is } \\
\text { trying to find } \\
\text { such books }\end{array}$ \\
\hline $\begin{array}{l}\text { Internet access } \\
\text { to websites } \\
\text { related to Harry } \\
\text { Potter }\end{array}$ & $\begin{array}{l}\text { Yes, to fulfill } \\
\text { the curiosity } \\
\text { about } \\
\text { alchemist } \\
\text { and volume } \\
7 \text { of Harry } \\
\text { Potter. }\end{array}$ & $\begin{array}{l}\text { No time to } \\
\text { access the } \\
\text { websites } \\
\text { associated } \\
\text { with Harry } \\
\text { Potter } \\
\text { because of } \\
\text { business at } \\
\text { school and } \\
\text { after school. }\end{array}$ & Never & $\begin{array}{l}\text { Yes, Harry } \\
\text { Potter.com }\end{array}$ & $\begin{array}{l}\text { Yes, but } \\
\text { stopped after } \\
\text { a while } \\
\text { because of } \\
\text { no new } \\
\text { information; } \\
\text { never } \\
\text { accessed } \\
\text { websites } \\
\text { related to } \\
\text { Wicca } \\
\text { (magic, } \\
\text { occult) }\end{array}$ \\
\hline \multicolumn{6}{|c|}{ Symbols of religions } \\
\hline $\begin{array}{l}\text { Harry Potter } \\
\text { offers a } \\
\text { particular } \\
\text { religious values / } \\
\text { doctrines }\end{array}$ & $\begin{array}{l}\text { Not offering } \\
\text { certain } \\
\text { religious } \\
\text { values, } \\
\text { generally } \\
\text { teaching not } \\
\text { to do black } \\
\text { magic. }\end{array}$ & Not at all & $\begin{array}{l}\text { Love, caring, } \\
\text { friendship. It } \\
\text { symbolizes } \\
\text { change, that } \\
\text { people have } \\
\text { many things } \\
\text { to change. }\end{array}$ & Not at all. & Not at all. \\
\hline $\begin{array}{l}\text { Harry Potter } \\
\text { texts and } \\
\text { Christian values }\end{array}$ & $\begin{array}{l}\text { Friendship } \\
\text { trust, the } \\
\text { difficulty of } \\
\text { living alone } \\
\text { without } \\
\text { friends and } \\
\text { family, the } \\
\text { challenges of } \\
\text { facing } \\
\text { difficulties } \\
\text { alone. }\end{array}$ & $\begin{array}{l}\text { Good- } \\
\text { hearted, } \\
\text { study hard, } \\
\text { smart, } \\
\text { understandin } \\
\text { g, } \\
\text { responsible, } \\
\text { not selfish, } \\
\text { obedient, } \\
\text { brave, not } \\
\text { greedy, } \\
\text { disciplined. }\end{array}$ & $\begin{array}{l}\text { No Christian } \\
\text { values in the } \\
\text { Harry Potter } \\
\text { text. }\end{array}$ & $\begin{array}{l}\text { No Christian } \\
\text { values in the } \\
\text { Harry Potter } \\
\text { text. }\end{array}$ & $\begin{array}{l}\text { No Christian } \\
\text { values in the } \\
\text { Harry Potter } \\
\text { text. }\end{array}$ \\
\hline $\begin{array}{l}\text { Christian } \\
\text { symbols in the } \\
\text { Harry Potter text }\end{array}$ & Not found & Not found & Not found & Not found & $\begin{array}{l}\text { With some } \\
\text { guidance the } \\
\text { respondent } \\
\text { can analyze } \\
\text { that if Harry } \\
\text { symbolizes } \\
\text { Jesus, then } \\
\text { Dumbledore } \\
\text { is God the } \\
\text { Father, and } \\
\text { Voldemort is } \\
\text { a demon. }\end{array}$ \\
\hline
\end{tabular}


Table2. Summary of Moral Values Identified by Respondents

\begin{tabular}{|c|c|c|c|c|c|}
\hline Moral Values & $\mathrm{R} 1$ & $\mathrm{R} 2$ & R3 & $\mathrm{R} 4$ & R5 \\
\hline There are good and bad sides in everyone & $\mathrm{X}$ & - & - & - & $\mathrm{X}$ \\
\hline Fairness & - & - & $\mathrm{X}$ & $\mathrm{X}$ & $\mathrm{X}$ \\
\hline Kindness & $\mathrm{X}$ & $\mathrm{X}$ & $\mathrm{X}$ & $\mathrm{X}$ & $\mathrm{X}$ \\
\hline Risk-taking & - & $\mathrm{X}$ & $\mathrm{X}$ & $\mathrm{X}$ & $\mathrm{X}$ \\
\hline Self-esteem & $\mathrm{X}$ & - & - & - & - \\
\hline Empathy & $\mathrm{X}$ & $\mathrm{X}$ & - & - & $\mathrm{X}$ \\
\hline Sportsmanship, can accept defeat / weakness with grace & - & - & - & - & $\mathrm{X}$ \\
\hline Knowledgeable / clever & $\mathrm{X}$ & $\mathrm{X}$ & $\mathrm{X}$ & $\mathrm{X}$ & $\mathrm{X}$ \\
\hline Open minded & - & - & - & $\mathrm{X}$ & - \\
\hline Principled & $\mathrm{X}$ & $\mathrm{X}$ & - & - & - \\
\hline Grateful & $\mathrm{X}$ & - & - & - & $\mathrm{X}$ \\
\hline Responsible & - & $\mathrm{X}$ & - & $\mathrm{X}$ & $\mathrm{X}$ \\
\hline Change, everyone can change into a better individual & - & - & $\mathrm{X}$ & - & $\mathrm{X}$ \\
\hline Wise & - & X & - & - & - \\
\hline Love for family & $\mathrm{X}$ & $\mathrm{X}$ & - & $\mathrm{X}$ & $\mathrm{X}$ \\
\hline Discipline & - & $\mathrm{X}$ & - & - & $\mathrm{X}$ \\
\hline Humorist & - & $\mathrm{X}$ & - & - & $\mathrm{X}$ \\
\hline Honest, having integrity & $\mathrm{X}$ & $\mathrm{X}$ & $\mathrm{X}$ & $\mathrm{X}$ & $\mathrm{X}$ \\
\hline Love for others, caring & $\mathrm{X}$ & $\mathrm{X}$ & $\mathrm{X}$ & $\mathrm{X}$ & $\mathrm{X}$ \\
\hline True compliance & $\mathrm{X}$ & $\mathrm{X}$ & - & - & - \\
\hline Leadership & - & $\mathrm{X}$ & - & - & - \\
\hline Trust in friends & $\mathrm{X}$ & - & - & $X$ & - \\
\hline Tenacity and perseverance & $\mathrm{X}$ & $\mathrm{X}$ & $\mathrm{X}$ & - & - \\
\hline Self-reliance & $\mathrm{X}$ & - & - & $\mathrm{X}$ & - \\
\hline Having a strong belief in something, not following along & $\mathrm{X}$ & - & - & $\mathrm{X}$ & - \\
\hline Optimistic & $\mathrm{X}$ & - & - & - & - \\
\hline Sensitive to the suffering of others & $\mathrm{X}$ & - & $\mathrm{X}$ & - & $\mathrm{X}$ \\
\hline Forgiving & - & - & - & $\mathrm{X}$ & - \\
\hline Self control & - & - & $\mathrm{X}$ & - & $\mathrm{X}$ \\
\hline Respect for the uniqueness of each individual & $\mathrm{X}$ & - & - & $\mathrm{X}$ & - \\
\hline Self confidence & $\mathrm{X}$ & - & - & - & $\mathrm{X}$ \\
\hline True friendship, solidarity & $\mathrm{X}$ & - & $\mathrm{X}$ & - & $\mathrm{X}$ \\
\hline Repentance & $\mathrm{X}$ & - & $\mathrm{X}$ & $\mathrm{X}$ & $\mathrm{X}$ \\
\hline Life choices & $\mathrm{X}$ & - & - & - & - \\
\hline Humble & $\mathrm{X}$ & - & - & - & $\mathrm{X}$ \\
\hline Patient & $\mathrm{X}$ & - & - & - & - \\
\hline Good deeds & $\mathrm{X}$ & - & - & $\mathrm{X}$ & - \\
\hline Serious & - & $\mathrm{X}$ & - & - & - \\
\hline Helpful & - & $\mathrm{X}$ & $\mathrm{X}$ & - & $\mathrm{X}$ \\
\hline Assertive & - & $\mathrm{X}$ & - & - & - \\
\hline Selfless & - & $X$ & - & - & $\mathrm{X}$ \\
\hline Not judging people just by their appearance & - & - & $\mathrm{X}$ & - & - \\
\hline Not greedy & - & $\mathrm{X}$ & - & - & $\mathrm{X}$ \\
\hline
\end{tabular}

All respondents can recognize the values contained in the Harry Potter text. The moral values mentioned by all respondents are kind, knowledgeable/talented, honest/having integrity, and love/care for others. Furthermore, ranked second and mentioned by four respondents is risk-taking, family love, and repentance. Six other values mentioned by each of the three respondents are fair, responsible, resilient, and perseverance, sensitive to the 
suffering of others, friendship, and helpful. Other values are mentioned randomly by one or two respondents. Once again, this confirms Matlin's theory that the same text can be interpreted differently by different text readers.

\section{Discussion}

The results of the study are summarized in the table below.

\begin{tabular}{|c|c|c|c|c|c|}
\hline & $\mathrm{R} 1$ & $\mathrm{R} 2$ & R3 & R4 & R5 \\
\hline Age & 13 & 13 & 13 & 11 & 12 \\
\hline Gender & Female & Female & Male & Female & Male \\
\hline Religiosity & Strong & Weak & Mediocre & Strong & Strong \\
\hline $\begin{array}{l}\text { The stage of } \\
\text { cognitive } \\
\text { development } \\
\text { according to } \\
\text { Piaget }\end{array}$ & $\begin{array}{l}\text { Beginning the } \\
\text { formal } \\
\text { operational } \\
\text { stage }\end{array}$ & $\begin{array}{l}\text { Beginning the } \\
\text { formal } \\
\text { operational } \\
\text { stage }\end{array}$ & $\begin{array}{l}\text { Beginning the } \\
\text { formal } \\
\text { operational } \\
\text { stage }\end{array}$ & $\begin{array}{l}\text { Concrete } \\
\text { operational } \\
\text { stage }\end{array}$ & $\begin{array}{l}\text { The end of } \\
\text { the concrete } \\
\text { operational } \\
\text { stage, the } \\
\text { beginning of } \\
\text { the formal } \\
\text { operational } \\
\text { stage }\end{array}$ \\
\hline $\begin{array}{l}\text { Discussion } \\
\text { forum at the } \\
\text { family / school }\end{array}$ & Yes & Almost none & No & Yes & Yes \\
\hline $\begin{array}{l}\text { The ability to } \\
\text { identify fiction } \\
\text { and facts }\end{array}$ & Capable & Capable & Capable & Capable & Capable \\
\hline $\begin{array}{l}\text { Violent } \\
\text { tendency }\end{array}$ & Not found & Not found & $\begin{array}{l}\text { Not found, } \\
\text { although } \\
\text { more likely to } \\
\text { prefer scenes } \\
\text { of violence } \\
\text { than female } \\
\text { respondents }\end{array}$ & Not found & $\begin{array}{l}\text { Not found, } \\
\text { although } \\
\text { more likely to } \\
\text { prefer scenes } \\
\text { of violence } \\
\text { than female } \\
\text { respondents }\end{array}$ \\
\hline $\begin{array}{l}\text { Occult } \\
\text { tendency }\end{array}$ & Not found & Not found & Not found & Not found & Not found \\
\hline $\begin{array}{l}\text { The ability to } \\
\text { realize } \\
\text { moral/noble } \\
\text { values in the } \\
\text { Harry Potter } \\
\text { texts }\end{array}$ & $\begin{array}{l}\text { Highly } \\
\text { capable }\end{array}$ & Capable & Capable & $\begin{array}{l}\text { Capable, but } \\
\text { at a lower } \\
\text { stage } \\
\text { compared to } \\
\text { other } \\
\text { respondents. }\end{array}$ & $\begin{array}{l}\text { Highly } \\
\text { capable }\end{array}$ \\
\hline
\end{tabular}

From the analysis of the results, all respondents aged 11-13 years were able to distinguish between fiction and fact. All respondents were able to sort out which of the Harry Potter texts were truly real and which were only imaginary.

Research shows that four out of five respondents admit to having tried at least one spell from the Harry Potter texts even though they had previously suspected that the spells in the Harry Potter texts werenot real. According to Piaget's theory of cognitive development, this is something normal because children in the concrete operational and formal operational stages generally show a remarkable capacity in logical reasoning and hypothetical reasoning. It means, with their reasoning skills, children form hypotheses about spells and test spells, then draw conclusions from the results of these experiments. 
Secondly, based on research conducted on the five respondents, no signs of a tendency towards occultism and violence are found. In both male respondents, namely R3 and R5, as is usually the case with boys, they prefer things that are more vulgar such as fighting scenes. However, there is no indication that both male and female respondents become violent after reading the Harry Potter texts. Likewise, there are no indications that the respondents know the occult. It is in agreement with Matlin's concept of perception, the inability of respondents to recognize this kind of occult symbol means that respondents do not have any background knowledge or pre-conception about the occult. As a result, respondents cannot interpret occult symbols even if they are in the Harry Potter texts. Thus, the concern that Harry Potter texts cause a tendency to Wicca and occultism to their readers is not proven in this research so far.

Thirdly, another component that has significant influences is the age of the respondent. Age affects the ability to comprehend the reading content and the respondent's maturity in responding to the texts, both in reasoning capability when making a distinction between fiction and fact and in understanding moral values.

Fourth, the availability of a forum that facilitates text discussion also has a more or less substantial influence on the understanding of the morals carried by the texts, as demonstrated by Respondent 1, who has such a forum in her family.

Fifth, this study has also found that the level of respondents' religiosity is directly proportional to their ability to identify moral values in the Harry Potter texts. Respondents with a high religious background are better able to automatically transfer the values of their religion when finding the moral in the texts.

\section{CONCLUSION AND SUGGESTION}

\section{Conclusions}

This study has found no strong evidence that the Harry Potter texts influence respondents to be sympathetic to the occult or to be violent. Jacques Derrida, in his deconstruction theory, said that a text is autonomous from its author. The writer is said to be dead, so he does not have the power to direct the interpretations of his readers. In other words, readers can interpret a text freely without having to refer to the author. In connection with this research, even if Rowling as the text writer includes elements of moral values of a particular religion or anyoccult elements, still Rowling cannot dictate the text reader to follow what she wrote.

This study has found that although respondents from both high and low religious backgrounds were able to identify moral values in the Harry Potter text, all respondents were not yet able to draw an analogy nor an allegory between the values of the text and their religion. The five respondents still thought in compartments; the Harry Potter texts and moral teachings in their religion or key figures in their lives were in different boxes. All respondents who were Christians were able to mention that the Harry Potter texts contained the values of love, sacrifice, self-control, selflessness, and integrity. Those are critical values in Christianity. None of the respondents, however, was able to draw an analogy between the text values and their religious values, even when given triggers. Only R5 could draw an analogy and make transfers.

Other interesting findings are related to the values identified by respondents to describe the characters in the text. Three respondents who were still in elementary school (R2, R3, and R4) mentioned many values related to the attributes of the International Baccalaureate (IB) Learner Profile, the international program implemented in the school when the study was conducted. 


\section{Suggestions}

Lately, student-centered learning has gained more and more attention. Constructivist methods in which learners explore and construct their own learning (self-construction of the meaning) are increasingly recognized as more effective learning methods because learning is more meaningful for the learners.

Likewise, moral value learning is not effective if it is through a teacher-centered method. Moral values taught through a teacher-centered approach make them a set of memorized packages that are unmeaningful to the learners. On the other hand, if moral value learning involves learners actively seeking, analyzing, and constructing meaning from their prior knowledge and new information, then surely the learning will be more engaging, challenging, and, most importantly, meaningful.

The stages of preparing for moral value learning through a constructivist approach are as follows:

1. Text selection

The selection of texts can be by a teacher/parent or an adult supervisor or by the learners themselves.

2. Text reading

Text can be read outside the classroom (as home reading) so as not to take up class time too much. If the entire class reads the same text, the teacher can facilitate by setting a reading schedule/target and dividing the text into some sections for more effective discussions.

3. Text discussion

This part is an essential part of the whole process. The teacher/parent/adult supervisor can facilitate the learning of moral values by providing critical and open-ended questions. If learners in a class read different books, teachers' questions can be more generic, such as:

a. Describe the main character.

b. What can you learn from the antagonist?

c. What can you learn from the protagonist?

Discussions can be in small groups with 3-4 students in a group, or a large group with the whole class discussing a subject. Small group discussions provide more opportunities for each learner to be more actively involved, while large group discussion can be used to clarify and reconfirm the learners' perceptions.

To ensure that each group member is active in small group discussions, teachers can monitor the students' participation in group discussions by drawing a diagram showing student interactions.

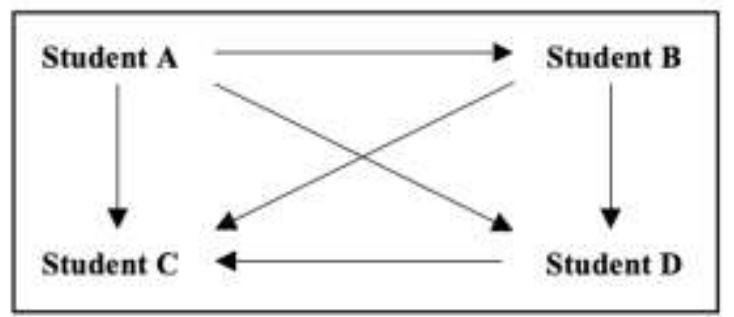

an example of an interaction diagram

Student A gives their opinions to Students B, C, and D.

Student B gives his opinion to Students C and D.

Student $\mathrm{C}$ does not give any comments.

Student D gives his opinion to Student C. 
4. Reflection

After the group discussion, each student has the opportunity to reflect on the moral values of the text for himself personally. It can be a written or oral assignment.

\section{REFERENCE}

Ashgate. (2011). Heroism in the Harry Potter Series.

Atkitson, Rita L., et al. (1996). Hilgard's Introduction to Psychology. Florida :Harcourt Brace \& Company.

Audifax. (2005). Mite Harry Potter: Psikosemiotika dan Misteri Simbol di BalikKisah Harry Potter.Yogyakarta \& Bandung: Jalasutra.

Asbari. M.,Nurhayati. W.,Purwanto.A,. (2020).The effect of parenting style and genetic personality on children character development. Jurnal Penelitian dan Evaluasi Pendidikan : 23(2). DOI: https://dx.doi.org/10.21831/pep.v23i2.28151

Asbari, M., Wijayanti,L.M, Hyun, C.C., Purwanto, A., Santoso, P.B,(2020).Effect of Tacit and Explicit Knowledge Sharing on Teacher Innovation Capability,Dinamika Pendidikan, 14(2),47-59,https://doi.org/10.15294/dp.v14i2.22732

Bettelheim, B. (1978). The Uses of Enchantment: The meaning and importance of fairy tale. New York: Vintage.

Bradley, E., Bell, S., Member, C., Jones, M.Nutter, J. H., \& Honors Director, D. (n.d.). (2019). Shaping Readers: The Moral Impact of Narrators.

Colbert, David. (2006). Magical World of Harry Potter: Dunia Ajaib Harry Potter: MitosMitos, Legenda-Legenda, dan Fakta-Fakta menakjubkan di Balik Karya Masterpiece ini. Jakarta: PT Gramedia Pustaka Utama.

Deaux, K., F.C Dane \& L.S. Wrightsman. (1993). Social Psychology in the 90 's.California: Brooks/Cole Publishing Company.

Dillistone, F.W. (2002). The Power of Symbols (Daya Kekuatan Simbol). Yogyakarta: Penerbit Kanisius.

Driyarkara. (1991). Driyarkara: tentang Pendidikan. Yogyakarta: Kanisius

Fraenkel, Jack R \& Wallen, Norman E. (1993). How to Design and Evaluate Research in Education. New York: McGraw-Hill.

Fraser, Lindsey. (2001). Conversation with J.K. Rowling. New York: Scholastic Inc.

Fudyartanto, Ki RBS. (2002). Psikologi Pendidikan dengan Pendekatan Baru. Yogyakarta: Global Pustaka Utama.

Gibb, N. (2003). The Real Magic of Harry Potter. Retrieved 13 December 2019, from https://time.com/4833705/the-real-magic-of-harry-potter/

Glasser, B. dan A.Strauss. (1967). The Discovery of Grounded Theory: Strategies for Qualitative Research. Chicago: Aldine, 1967.

Goddard, Andrew. Harry Potter and the Quest for Virtue Harry Potter and the Quest for Virtue.Anvil Volume 18 No 3. (2001) 
Hardiman, F. Budi. (2003). Melampaui Positivisme dan Modernitas. Yogyakarta: Kanisius.

Hardiman, F. Budi. (1992). Penelitian dan Praksis. Dua Paradigma Penelitian dalam Perspektif Teori Kritis dalam Gema Duta Wacana, no. 42.

Houghton, John. (2007). The Harry Potter Effect: Simbol Kekristenan atau Kegelapan? (judul asli: A Closer Look at Harry Potter). Yogyakarta: Penerbit Andi.

Kern, Edmund M. (2006). The Wisdom of Harry Potter: Pelajaran Moral dari Pahlawan Favorit Kita. Jakarta: PT Gramedia Pustaka Utama.

Kjos, B. (2007). Harry's Last Battles \& Rowling's Beliefs. Retrieved 13 December 2019, fromhttp://www.crossroad.to/articles2/007/harry-deathly.htm

Kjos, B. (2005). Harry Potter and the Half-Blood Prince. Retrieved 13 December 2019, from http://www.crossroad.to/articles2/05/half-blood.htm

Kjos, B. (2003). Harry Potter \& The Order of the Phoenix: "It's only fantasy"and other deceptions". $\quad$ Retrieved 13 December 2019, from http://www.crossroad.to/articles2/2003/phoenix.htm

Kjos, B. (2001). Twelve reasons not to see Harry Potter movies. Retrieved 13 December 2019, from http://www.crossroad.to./articles2/HP-Movie.htm

Lanz, Peter. (2003). Harry Potter: The story behind the stories. Retrieved 13 December 2019, from http://crossroad.to/ask-peter/hp-overview-1.htm.

Literature, Psychoanalysis, and the Re-Formation of the Self: A New Direction for ReaderResponse Theory. (n.d.). https://doi.org/10.2307/462087

Matlin, Margaret W. (1994). Cognition. Orlando: Harcourt Brace Publisher.

Nasution, S. (1998). Penelitian Naturalistik Kualitatif. Bandung: Tarsito.

Neal, Connie. (2007). Injil Menurut Harry Potter: Spiritualitas dalam Kisah Seeker yang Paling Terkenal Sedunia. (judul asli: The Gospel Accordingto Harry Potter) Jakarta: PT BPK Gunung Mulia.

Piaget, J., \& Inhelder, B. (1969). The Psychology of the Child. New York: BasicBooks.

Rice, F. Philip. (1999). The Adolescent: Development, Relationship, and Culture. Boston: Allyn and Bacon.

Rowling, J.K. (1999). Harry Potter and the Chamber of Secrets. New York: Scholastic Inc.

Rowling, J.K. (2007). Harry Potter and the Deathly Hallows. New York: Scholastic Inc.

Rowling, J.K. (2000). Harry Potter and the Goblet of Fire. London: Bloomsbury Publishing Plc.

Rowling, J.K. (2005). Harry Potter and the Half Blood Prince. London: Bloomsbury Publishing Plc.

Rowling, J.K. (1997). Harry Potter and the Philosopher's Stone. London:Bloomsbury Publishing Plc.

Rowling, J.K. (1999). Harry Potter and the Prisoner of Azkaban. New York: Scholastic Inc. 
Rowling, J.K. (2004). Harry Potter Dan Orde Phoenix. Jakarta: PT. Gramedia Pustaka Utama.

Rowling, J. (2002). Quidditch dari masa ke masa. Jakarta: Gramedia.

Scamander, Newt. (2002). Fantastic Beasts \& Where to Find Them: Hewan-Hewan Fantastis dan Di Mana Mereka Dapat Ditemukan. Jakarta: Gramedia Pustaka Utama.

Bradley, Emily. (2019). Shaping Readers: The Moral Impact of Narrators. (n.d.). Retrieved fromhttps://digitalcommons.liberty.edu/cgi/viewcontent.cgi? article=1894\&context= honors

Sudiarja, A. (2007, Juli-Agustus). Driyarkara: Pendidikan Kepribadian Nasional. Basis, 0708, Tahun ke-56.

Suharnan. (2005). Psikologi Kognitif. Surabaya: Penerbit Srikandi.

Sumaryono. (1999). Hermeneutik: Sebuah Metode Filsafat. Yogyakarta: Penerbit Kanisius.

Waters, G. and Mithrandir, A. (2003). Ultimate unofficial guide to the mysteries of Harry Potter: Analysis of Book 1-4. Niles, IL: Wizarding World Press.

Waters, G. and Mithrandir, A. (2006). Ultimate unofficial guide to the mysteries of Harry Potter: Analysis of Book 5. Niles, Ill.: Wizarding World.

Weston, J. (2017). FireScholars Finding faith in fantasy: exploring the chronicles of Narnia, Harry Potter, and His Dark Materials. Retrieved fromhttps://firescholars.seu.edu/honors

Wolf, A. (n.d.). ScholarlyCommons HarryPotter and the Enhancement of Hope: What Harry Potter and Positive Psychology can Teach Us about the Good Life. Retrieved from https://repository.upenn.edu/mapp_capstonehttps://repository.upenn.edu/mapp_caps tone/156 